\title{
La mobilisation des acteurs régionaux face à la migration des jeunes : le cas de la Stratégie MigrAction au Saguenay-Lac-St-Jean (Québec)
}

\author{
Hervé Stecq ${ }^{1}$ \\ Université du Québec à Chicoutimi
}

\section{INTRODUCTION}

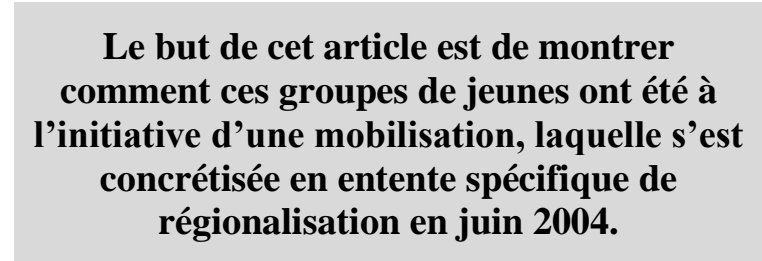

L'enjeu de migration des jeunes a suscité une forte mobilisation des acteurs régionaux au Saguenay-Lac-Saint-Jean, depuis le début des années 2000. En effet, le solde migratoire chez les jeunes de moins de 35 ans s'est dégradé au cours de ces trente dernières décennies, au point que l'exode a été comparé à une «hémorragie » ou une «saignée ». En 2003, des groupes de jeunes ont décidé de «passer en mode solution » pour résorber un phénomène ayant de graves conséquences sur le développement régional.

Le but de cet article est de montrer comment ces groupes de jeunes ont été à l'initiative d'une mobilisation, laquelle s'est concrétisée en entente spécifique de régionalisation en juin 2004. Un autre des objectifs est de prouver que la mobilisation régionale est en réalité l'activation de réseaux latents.

Tout d'abord, dans cet article, sera constaté le phénomène de la migration des jeunes au Saguenay-Lac-Saint-Jean, à l'aide notamment de données statistiques de l'Institut de la Statistique du Québec (ISQ). Des explications seront apportées sur le phénomène, à savoir son origine historique et ses causes multiples. Ensuite sera décrite la Stratégie MigrAction, une stratégie globale élaborée en 2003 dans l'espoir de rétablir un bilan migratoire positif au Saguenay-LacSaint-Jean. Enfin, seront relatées les phases de mobilisation autour de l'enjeu de migration des jeunes, phases qui révèleront l'existence, puis la mobilisation d'un réseau d'acteurs régionaux.

\section{LE PHÉNOMÈNE DE LA MIGRATION DES JEUNES AU SAGUENAY-LAC-ST-JEAN}

\subsection{Constat d'un phénomène}

Avec un solde migratoire de +3 entre 2010 et 2011, le Saguenay-Lac-Saint-Jean compte parmi les régions ayant un faible potentiel d'attraction ${ }^{2}$. Faisant partie de ce cercle, sont les régions ayant un solde migratoire négatif ou à peine positif : Montréal (- 22 207), Nord-duQuébec (-154), Bas-Saint-Laurent (-28), CôteNord (+ 37), Abitibi-Témiscamingue (+ 53). Les chiffres de Montréal doivent être cependant relativisés, compte tenu qu'ils sont marginaux par rapport à la population totale.
Une étude du solde migratoire du Saguenay-LacSaint-Jean par tranche d'âge, pour la même période, révèle qu'il est négatif pour les : 10-14 ans (- 3), 15-19 ans (- 31), 20-24 ans (- 372), 7579 ans (-21) et 80 ans et plus (-12) (figure 1). La tranche des 20-24 ans est particulièrement déficitaire. S'agit-il d'un constat passager, ou bien d'un phénomène installé dans la durée?

Une étude du solde migratoire du Saguenay-Lac-Saint-Jean par tranche d'âge, pour la même période, révèle qu'il est négatif. 
Figure 1 - Solde migratoire interrégional selon le groupe d'âge, Saguenay-Lac-St-Jean, 2010-2011

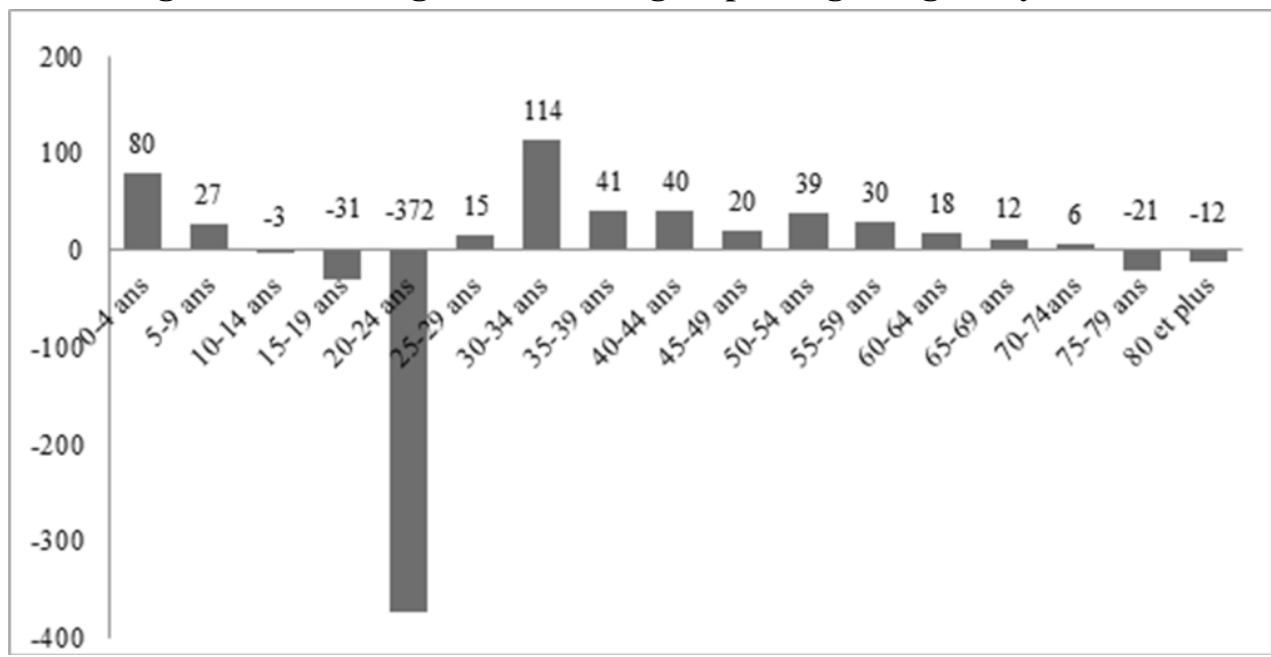

Source : ISQ, exploitation du Fichier d'inscription des personnes assurées (FIPA) de la Régie de l'assurance maladie du Québec (RAMQ).

Des données plus précises font état d'un solde migratoire négatif pour toutes les catégories, au cours de périodes : 1991-1996, 1996-2001, 20012006 (figure 2). Cependant, il est de loin le plus déficitaire pour les catégories de 20-24 ans et de 2529 ans. De 1996 à 2006, le taux net de la migration interrégionale pour ces dernières, était en moyenne de $-16,5 \%$, alors qu'il était de $-5 \%$ pour les 30 34 ans, troisième catégorie la plus déficitaire. À la lumière de ces chiffres, il convient de mentionner l'existence d'un phénomène de migration chez les jeunes, au Saguenay-Lac-Saint-Jean.

Figure 2 - Migration interrégionale quinquennale par groupe d'âge, Saguenay-Lac-St-Jean, 1991-1996, 1996-2001 et 2001-2006

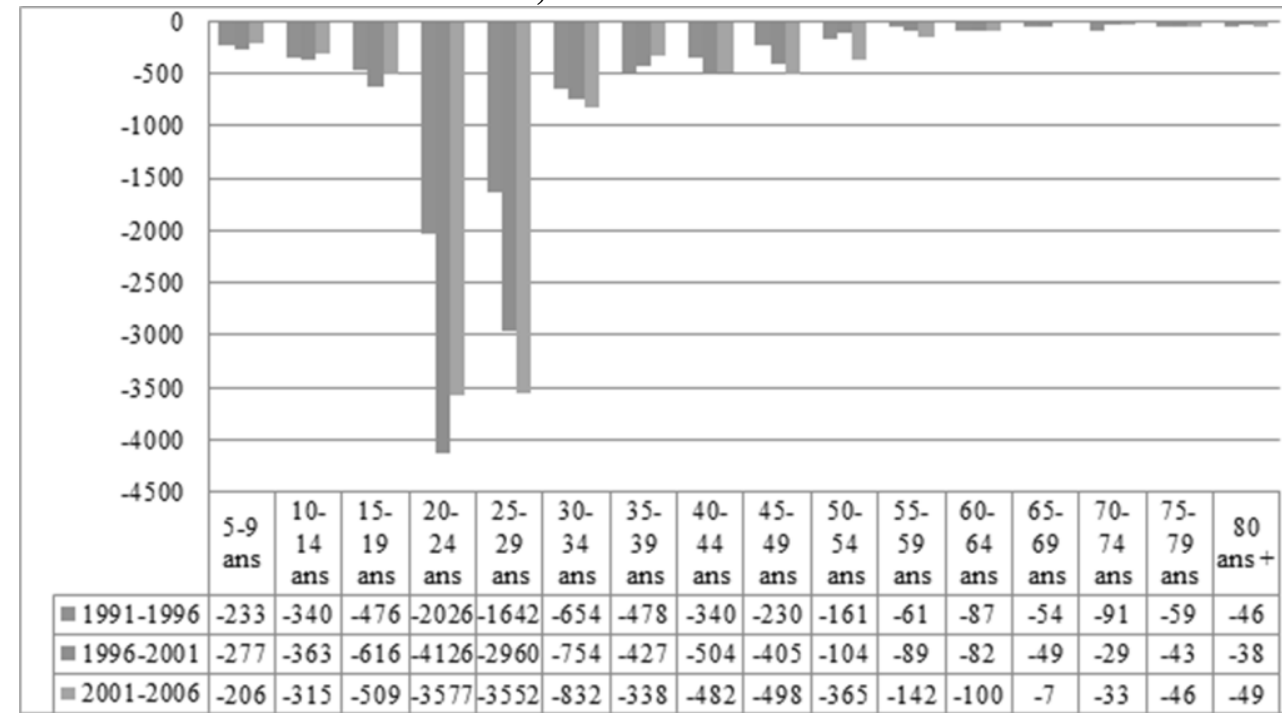

Source : ISQ (2012)

\subsection{Un phénomène de longue date}

Jusqu'aux années 1960, des taux de natalité élevés, associés à des taux de mortalité relativement bas, ont assuré un certain accroissement des populations régionales.
Le phénomène de migration des jeunes du Saguenay-Lac-Saint-Jean n'est pas récent; il est lié à l'histoire des migrations de la région. Il fut qualifié d'exode en raison des faibles retours. Dès la fin du XVIII ${ }^{e}$ siècle, le Saguenay-Lac-Saint-Jean offre un bilan migratoire mitigé. Au XIX ${ }^{\mathrm{e}}$ siècle, alors que le 
Lac-Saint-Jean accueille des immigrants, le Saguenay voit une partie de sa population émigrer. $\mathrm{Au} \mathrm{XX} \mathrm{X}^{\mathrm{e}}$ c'est l'inverse qui se produit ${ }^{5}$. Jusqu'aux années 1960, des taux de natalité élevés, associés à des taux de mortalité relativement bas, ont assuré un certain accroissement des populations régionales, cela malgré des soldes migratoires généralement négatifs. Le problème ne se manifeste qu'à partir des années 1980, avec une chute du taux de natalité en deçà du seuil de renouvellement des générations ${ }^{2}$.

\subsection{Les causes du phénomène}

\section{Progressivement, les pistes de recherche se sont orientées sur des facteurs socioculturels.}

Le phénomène migratoire a attiré l'attention des chercheurs, car ses causes étaient encore très incertaines. Effectivement, l'emploi est vite apparu comme un facteur principal de migration. Mais comment expliquer que des jeunes du secondaire encore peu concernés par leur insertion professionnelle, émettent autant le désir de migrer ${ }^{3}$ ? Ce phénomène serait-il plurifactoriel? Toujours estil que plusieurs recherches ont été réalisées au Saguenay-Lac-Saint-Jean afin de mieux cerner les facteurs intervenant dans la décision des jeunes de migrer. L'année 1999 annonce le début d'une ère prolifique quant au thème de la migration des jeunes, alors qualifié d'exode.

Les premières recherches menées ont conforté la croyance générale selon laquelle cette migration était principalement liée à des problématiques d'insertion professionnelle ${ }^{6}$. Pour d'autres, elle était la conséquence d'un manque d'investissements publics ou d'un dysfonctionnement du système de financement du gouvernement du Québec ${ }^{7}$. Progressivement, les pistes de recherche se sont orientées sur des facteurs socioculturels. Certains jeunes quitteraient la région pour aller se former ${ }^{8}$, d'autres pour « vivre leur vie »"

\subsection{L'attractivité comme enjeu de développement du Saguenay-Lac-Saint-Jean}

De ce portrait démographique du Saguenay-LacSaint-Jean, peut être dressé un constat de faible attractivité en raison du bilan migratoire à peine positif. Ce constat est d'autant plus vrai pour les jeunes de 20 à 24 ans. Le rétablissement d'un bilan migratoire, notamment chez les jeunes, apparaît dès lors comme un des principaux enjeux pour le Saguenay-Lac-Saint-Jean. Très sommairement, le solde migratoire exprime la différence entre les entrées et les sorties. Il sera positif quand ces premières seront supérieures à ces dernières, et inversement négatif. Les facteurs d'attractivité seront donc : le retour, l'attraction et la rétention des populations. Ils seront in fine des défis à relever pour rétablir un solde migratoire positif.

\section{Le rétablissement d'un bilan migratoire, notamment chez les jeunes, apparaît dès lors comme un des principaux enjeux pour le Saguenay-Lac-Saint-Jean.}

\section{LA STRATÉgIE MIGRACTION : UNE STRATÉgIE GLOBALE POUR RÉTABLIR UN BILAN MIGRATOIRE POSITIF CHEZ LES JEUNES}

\subsection{Définition et présentation de la Stratégie MigrAction}

La Stratégie MigrAction, aussi appelée «entente spécifique visant à influencer positivement le bilan migratoire des jeunes au Saguenay-Lac-SaintJean », fut signée le 25 juin 2004 pour une durée de trois ans. Par cette entente, quinze partenaires (CRÉ, organisations de jeunesse, MRC) s'engageaient à prendre des mesures pour contrer les effets négatifs induits par le phénomène de migration des jeunes. Cependant, la Stratégie MigrAction fut initialement créée le 15 septembre 2003 à l'initiative de groupes et d'organismes de jeunesse du Saguenay-LacSaint-Jean, en l'occurrence le Regroupement action jeunesse-02 (RAJ-02) et les trois Carrefours jeunesse emploi (CJE) de la région. Le renouvellement de l'entente spécifique a été officialisé le 14 juillet 2008, avec 36 partenaires issus de l'éducation (Commissions scolaires du Saguenay-Lac-SaintJean, cégeps), de la finance (Desjardins), de l'insertion à l'emploi (CLE), etc. Il s'agit donc d'un cas réussi de mobilisation d'acteurs régionaux.

La stratégie MigrAction est dite globale en raison de la transversalité de ses axes d'intervention, à la fois dans le domaine économique, social et culturel. 
La stratégie MigrAction est dite globale en raison de la transversalité de ses axes d'intervention, à la fois dans le domaine économique, social et culturel. Les causes de ce phénomène étant multiples, les solutions seront logiquement variées. La Stratégie MigrAction sera alors déclinée en cibles d'intervention : 1) garantir une image positive de la région; 2) implanter des mesures incitatives à l'établissement; 3) aider à l'insertion socioprofessionnelle; 4) développer la culture entrepreneuriale; 5) favoriser l'implication sociale des jeunes.

\subsection{Le contexte québécois de régionalisation des politiques de migration des jeunes}

La Stratégie MigrAction se comprend mieux à la lumière du contexte québécois de prise en charge de la question de migration des jeunes. Dans les politiques de jeunesse successives, le gouvernement du Québec a rappelé l'importance de maintenir les jeunes dans leur milieu, dans le souci de pérenniser un développement régional. $\mathrm{La}$ migration des jeunes contient des enjeux territoriaux et sociaux, compte tenu des risques de dévitalisation de certaines localités. La Stratégie MigrAction fut mise sur pied en 2004 comme une réponse régionale aux requêtes du Plan d'action jeunesse 2002-2005 ${ }^{10}$. Elle fut renouvellée en 2008 , en accord avec les objectifs formulés dans la Stratégie d'action jeunesse 2006-2009 ${ }^{11}$ : «favoriser le maintien, le retour et l'établissement des jeunes en région. »

\section{La Stratégie MigrAction fut mise sur pied en 2004 comme une réponse régionale aux requêtes du Plan d'action jeunesse 2002-2005.}

\section{LA STRATÉGIE MIGRACTION : UNE MOBILISATION DE RÉSEAUX LATENTS}

3.1 Les réseaux des organisations jeunesse du Saguenay-Lac-Saint-Jean (2001-2004)

Bien avant la création de la Stratégie MigrAction, le RAJ-02 et les CJE avaient consolidé leurs réseaux grâce à des actions collectives destinées aux jeunes. Les plus structurantes ont été celles consacrées à l'insertion professionnelle des jeunes, compte tenu de l'ampleur des liens alors tissés entre acteurs locaux ou régionaux. Enfin, des comités de travail ont démontré leur potentiel rassembleur à une échelle régionale. L'ensemble de ces événements témoigne de l'existence de réseaux de collaboration. À de nombreuses occasions, plusieurs acteurs sont rentrés en interactions pour concrétiser des projets inhérents aux problématiques de jeunesse, que ce soit pour l'insertion professionnelle, la sensibilisation ou la concertation en vue de partenariats futurs. Cet enchevêtrement de relations peut être graphiquement représenté (figure 3).

Figure 3 - Réseau de coopération du RAJ-02, du comté Roberval, du CJE Lac-Saint-Jean Est et du CJE Saguenay (2001-2004)

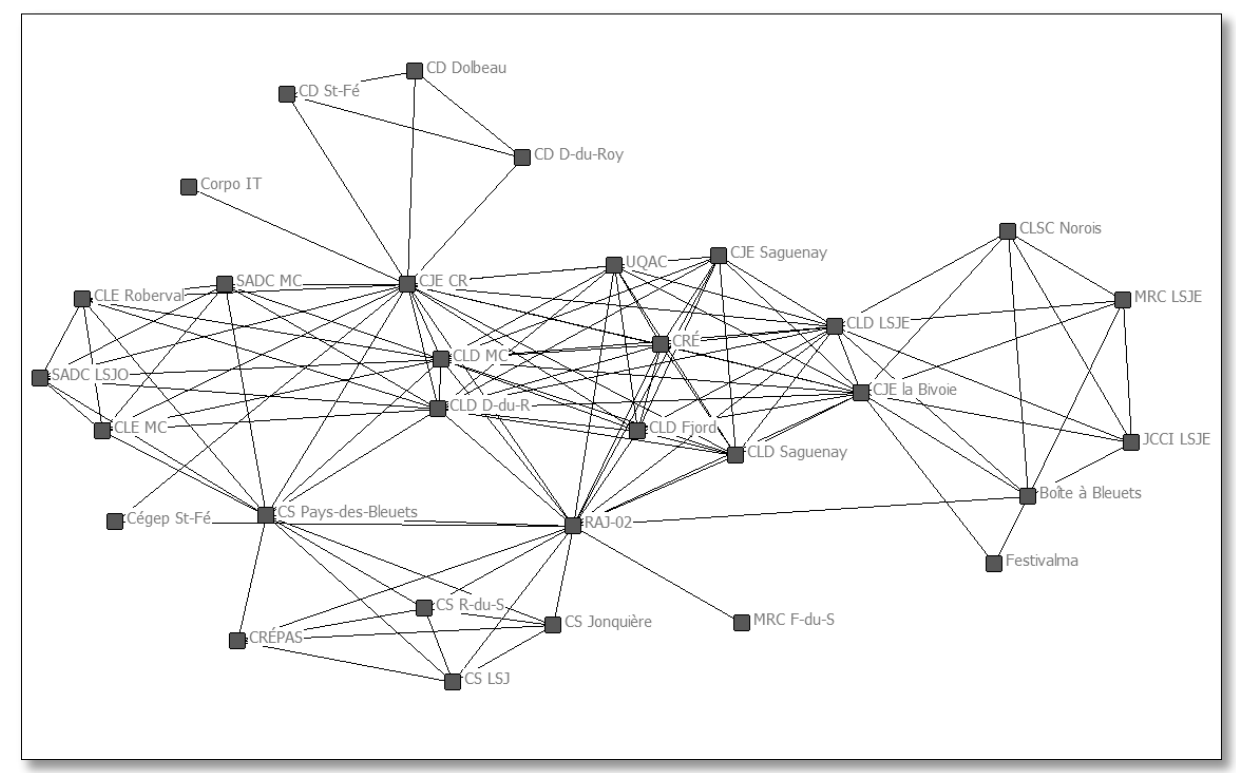




\subsection{Des réseaux en mobilisation (2001-2011)}

\section{L'étude de la Stratégie MigrAction a permis de mettre en exergue des éléments nécessaires à la réalisation des étapes succinctes de mobilisation régionale.}

Ces réseaux de coopération ont été mobilisés par phases, entre 2001 et 2011. L'étude de la Stratégie MigrAction a permis de mettre en exergue des éléments nécessaires à la réalisation des étapes succinctes de mobilisation régionale. Avaient été distinguées plusieurs phases: de diagnostic, de mobilisation initiale, de vision commune et de planification des actions collectives d'opérationnalisation, d'évaluation et de renouvellement.

Dans la phase de diagnostic (2001), la migration des jeunes a été plébiscitée comme étant un problème à échelle régionale. La phase de mobilisation initiale (2002-2003) était celle de l'identification d'activités mobilisatrices et de partenaires potentiels. La dense phase de vision commune et de planification des actions collectives (2003-2004), faisait ressortir trois éléments : 1) la définition de cinq cibles d'intervention constituait un consensus; 2) le financement d'envergure régionale; 3) la dotation d'indicateurs communs. La phase d'opérationnalisation (2004-2007) passait par la réalisation d'actions mobilisatrices, permettant à un grand nombre d'acteurs, de localités et secteurs différents, de pouvoir s'associer. Dans la phase d'évaluation et de renouvellement (2007-2008), deux éléments ont été notés : 1) une culture de l'évaluation et des compétences est requise pour le déroulement de cette phase; 2) les agents mobilisateurs doivent avoir la capacité de persuader les bailleurs de fonds de renouveler leur participation au partenariat. Suivra enfin une phase de deuxième opérationnalisation (2008-2011).

\subsection{Des réseaux en structuration}

\section{Après 2004, d'autres acteurs régionaux ont} été mobilisés.

Si les étapes de la mobilisation ont surtout consisté à mobiliser un réseau, elles n'ont pas moins été structurantes depuis 2004 à $2011^{12}$. Cette structuration aurait à son tour créé la condition même de la mobilisation, dans la mesure où l'existence préalable de réseaux sociaux serait une condition sine qua non à toute mobilisation. Après 2004, d'autres acteurs régionaux ont été mobilisés. Ce serait là un cercle vertueux de la mobilisation.

Les réseaux du RAJ-02 et des CJE se sont élargis depuis la signature de la première entente spécifique en 2004. Des organisations locales (Chambres du commerce et de l'industrie) et régionales (CRÉPAS, Centre de solidarité international), ainsi que des entreprises (Bell, Canal Vox, Malette, etc.), se sont jointes à un réseau principalement constitué d'organismes publics et de collectivités territoriales. Le changement notable après 2004, est certainement l'inclusion de plusieurs entreprises locales ou régionales telles que Malette, Unibec ou Ferlac. Le réseau se serait de fait diversifié en incluant des partenaires et collaborateurs de toutes les sphères d'acteurs régionaux (figure 4).

Figure 4 - Réseau de coopération du RAJ-02 et des CJE du Saguenay-Lac-Saint-Jean (2001-2011)

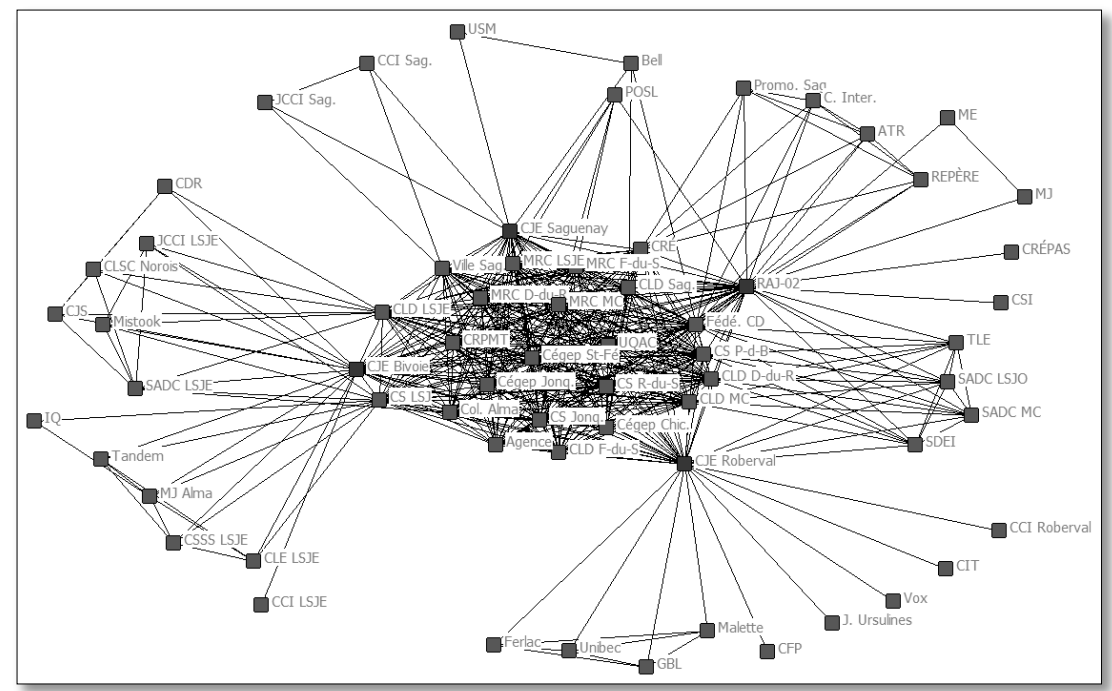


Cette structuration pourrait avoir été le fait des CJE. Ils continuent d'être des relais entre leur localité respective et le reste de la région, par le biais de la Stratégie MigrAction, ce qui perpétuerait la mobilisation régionale. Le CJE La Bivoie partage cette fonction avec le CLD LacSaint-Jean-Est et la Commission scolaire du LacSaint-Jean, contribuant à densifier les liens dans la MRC Lac-Saint-Jean-Est. Les CJE Saguenay et

\section{CONCLUSION}

\section{La Stratégie MigrAction serait la preuve que les stratégies globales ont un grand potentiel mobilisateur.}

La migration des jeunes s'avère de plus en plus être un enjeu d'attractivité territoriale. Outre le Saguenay-Lac-Saint-Jean, des régions périphériques telles que le Bas-Saint-Laurent, l'AbitibiTémiscamingue et la Gaspésie-Île-de-laMadeleine, devront relever le défi de l'attraction et de la rétention des jeunes. D'aucuns pensent qu'elles sont aujourd'hui en compétition pour ravir le nombre toujours plus important de jeunes désirant s'établir en région.

La Stratégie MigrAction serait la preuve que les stratégies globales ont un grand potentiel mobilisateur. Le nombre d'actions collectives qu'elles génèrent dans des axes variés, sont en effet susceptibles de regrouper un grand nombre d'acteurs régionaux. Cela pourrait bien expliquer le succès de la Stratégie MigrAction qui, en l'espace de 4 ans, passe de 15 à 36 partenaires. Enfin, cet exemple démontre bien que mobilisation n'est pas seulement l'activation de réseaux latents; elle est aussi structurante. Des réseaux s'étoffent alors que des acteurs mènent des actions conjointement.

\section{BIBLIOGRAPHIE ET NOTES}

${ }^{1}$ Hervé Stecq est diplômé du doctorat en développement régional, offert conjointement par l'Université du Québec à Chicoutimi (UQAC) et par l'Université du Québec à Rimouski (UQAR). Il est actuellement chargé de cours à l'UQAC.

${ }^{2}$ Données de l'ISQ, exploitation du Fichier d'inscription des personnes assurées (FIPA) de la Régie de l'assurance maladie du Québec (RAMQ). comté Roberval sont les principaux mobilisateurs locaux intervenant en matière de bilan migratoire chez les jeunes. Le RAJ-02 quant à lui, confirme son statut d'organisme régional en structurant son réseau autour d'organisations ayant une assise régionale, ou d'autres organismes dans différentes MRC. De ce point de vue, l'enjeu de migration aura été un motif de structuration, donc un élément rassembleur.

${ }^{3}$ Pouyez, C. et al. (1983). Les Saguenayens : introduction à l'histoire des populations du Saguenay, $\mathrm{XVI}^{\mathrm{e}}-\mathrm{XX}^{\mathrm{e}}$ siècles, Québec, PUQ, $386 \mathrm{p}$.

${ }^{4}$ Leblanc, P., C. Girard, S. Côté et D. Potvin (2003). «La migration des jeunes et le développement régional dans le croissant péri-nordique du Québec », Recherches sociographiques. La migration des jeunes, vol. 44, no 1, p. 35-55.

${ }^{5}$ Gaudreault, M., M. Perron, S. Veillette et L. Richard (2000). Rester, partir ou revenir au Saguenay - LacSaint-Jean après les études. Analyse du désir d'enracinement des élèves du secondaire, Jonquière, Cégep de Jonquière-Groupe ÉCOBES, 67 p.

${ }^{6}$ Cartier, F. (2000). Les carrières d'avenir au Québec, Québec, Éditions Ma Carrière, $417 \mathrm{p}$.

${ }^{7}$ Côté, C. et D. Larouche (2000). Radiographie d'une mort fine: dimension sociale de la maladie au Québec, Chicoutimi, JCL, 286 p.

${ }^{8}$ Simard, M., S. Ouellet, V. Fortin et S. Tremblay (2006). Cadres de vie et intentions migratoires des jeunes à Saguenay, Chicoutimi, Groupe de recherches et d'interventions régionales (GRIR), $81 \mathrm{p}$.

${ }^{9}$ Gauthier, M. (2004). «À la recherche du «sens » de la migration des jeunes Québécois », dans P. LeBlanc et M. Molgat (dir.), La migration des jeunes. Aux frontières de l'espace et du temps, Sainte-Foy, PUL, p. 5-32.

${ }^{10}$ Secrétariat à la jeunesse (2002). Plan d'action jeunesse 2002-2005. La jeunesse au cœur du Québec, Québec, Gouvernement du Québec, 52 p.

${ }^{11}$ Secrétariat à la jeunesse (2006). Pour une jeunesse engagée dans sa réussite. Stratégie d'action jeunesse 2006-2009, Québec, Gouvernement du Québec, p 39. ${ }^{12}$ Près de $70 \%$ des partenaires régionaux de la deuxième entente spécifique MigrAction signée en 2008, faisait déjà partie du même réseau de coopération à la veille de la conclusion de la première entente du 25 juin 2004. 